\title{
Halichoblelide D, a New Elaiophylin Derivative with Potent Cytotoxic Activity from Mangrove-Derived Streptomyces sp. 219807
}

\author{
Ying Han ${ }^{\dagger}$, Erli Tian ${ }^{\dagger}$, Dongbo Xu, Min Ma, Zixin Deng and Kui Hong * \\ Key Laboratory of Combinatorial Biosynthesis and Drug Discovery, Ministry of Education, \\ School of Pharmaceutical Sciences, Wuhan University, Wuhan 430071, China; hanying0928@whu.edu.cn (Y.H.); \\ 2014103060011@whu.edu.cn (E.T.); xudongbo@whu.edu.cn (D.X.); winder_568@163.com (M.M.); \\ zxdeng@whu.edu.cn (Z.D.) \\ * Correspondence: kuihong31@whu.edu.cn; Tel./Fax: +86-27-6875-2442 \\ + These authors contributed equally to this work. \\ Academic Editor: Derek J. McPhee \\ Received: 23 May 2016; Accepted: 21 July 2016; Published: 25 July 2016
}

\begin{abstract}
During our search for interesting bioactive secondary metabolites from mangrove actinomycetes, the strain Streptomyces sp. 219807 which produced a high elaiophylin yield of $4486 \mathrm{mg} / \mathrm{L}$ was obtained. A new elaiophylin derivative, halichoblelide D (1), along with seven known analogues 2-8 was isolated and identified from the culture broth. Their chemical structures were determined by detailed analysis of 1D and 2D NMR and HRMS data. The absolute configuration of halichoblelide D (1) was confirmed by comparing the CD spectrum with those of the reported analogues. Compounds 1-7 exhibited potent cytotoxic activities against HeLa and MCF-7 cells with $\mathrm{IC}_{50}$ values ranging from 0.19 to $2.12 \mu \mathrm{M}$.
\end{abstract}

Keywords: mangrove actinomycetes; Streptomyces sp. 219807; elaiophylin; cytotoxic

\section{Introduction}

There is a continuous and urgent demand for iterative cycles of drug discovery and development to combat new emerging human diseases and multi-drug resistant pathogens [1,2]. Microbial natural products have delivered a plethora of structurally diverse specialized metabolites with potent pharmaceutical properties [3]. Microorganisms derived from underexplored habitats, such as mangrove ecosystems, are especially noteworthy, in part owing to their unique biosynthetic potential and the corresponding attenuation of the likelihood of finding high numbers of known compounds [4-6]. Mangrove ecosystems are extensively distributed in the interface between terrestrial and marine environments at tropical and subtropical latitudes, thereby suffering from extremely harsh stresses, including temperature, salinity, moisture and anoxia [7]. Therefore, mangrove-derived actinomycetes are distinct from terrestrial isolates in physiology and genotype to cope with the rigorous environments. Several decades of studies have demonstrated that actinomycetes recovered from mangrove systems are increasingly recognized as a vast reservoir of new bioactive natural products, such as salinosporamide A [8], xiamycins [9], and streptocarbazoles A and B [10]. Accordingly, our studies are focused on the mangrove ecosystem actinomycetes.

After an initial cytotoxic screening of fermentation extracts of actinomycetes isolated from mangrove soil collected in Sanya, the active strain Streptomyces sp. 219807 was selected for further investigation. By means of HRMS match in the AntiBase 2012, elaiophylin and many unassigned elaiophylin-related compounds were successfully detected in the ethyl acetate extract of the fermentation broth. Elaiophylins are a family of glycosylated 16-membered macrolides featuring C2 symmetry produced 
by several Streptomyces spp. [11-15], which exhibit remarkable biological properties, such as antibacterial [16,17], anthelminthic [18], anticancer [19], antiviral [20] and immunosuppressant activities [21]. Chemical investigation of the bioactive extract led to the discovery of eight elaiophylins including a new derivative, halichoblelide $\mathrm{D}(\mathbf{1})$, together with 2-methyl-11,11'-O-dimethylelaiophylin (2), 2-methylelaiophylin (3), elaiophylin (4), 11-O-methyl-elaiophylin (5), 11,11'-O-dimethylelaiophylin (6), efomycin G (7) and 11',12'-dehydroelaiophylin (8) (Figure 1). Details of their isolation, structure elucidation and bioactivities are reported herein.
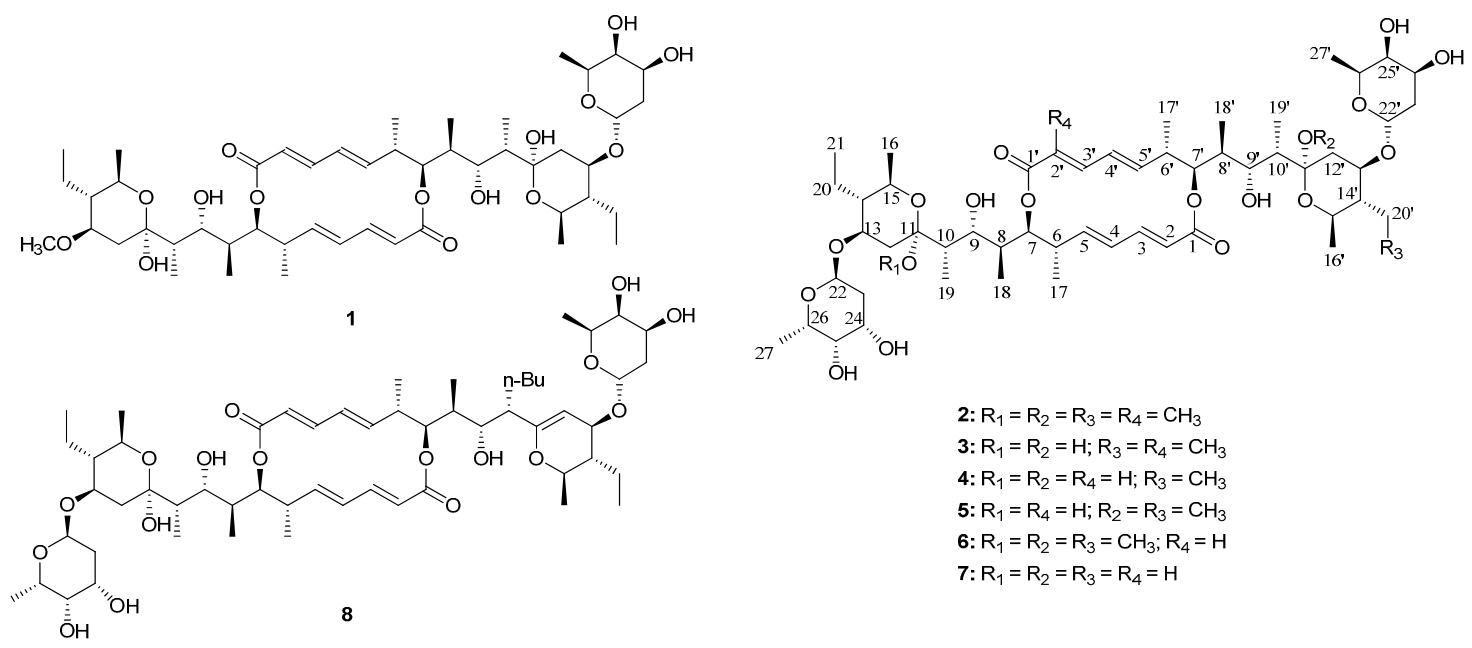

Figure 1. Chemical structures of compounds 1-8.

\section{Results}

\subsection{Structure Elucidation}

Halichoblelide D (1) was obtained as a white needle-like crystals. Its molecular formula was determined as $\mathrm{C}_{49} \mathrm{H}_{80} \mathrm{O}_{15}$ by the negative HRESIMS at $m / z 943.5183\left[\mathrm{M}+\mathrm{Cl}^{-}\right.$(calc. for $\mathrm{C}_{49} \mathrm{H}_{80} \mathrm{O}_{15} \mathrm{Cl}^{-}$, 943.5186), implying 10 degrees of unsaturation. The UV $(252 \mathrm{~nm})$ and $\operatorname{IR}\left(1699,1636 \mathrm{~cm}^{-1}\right)$ absorption indicated the presence of an aliphatic conjugated ester system in the molecule [22]. A close inspection of the ${ }^{1} \mathrm{H}-\mathrm{NMR}$ (Table 1 ) and ${ }^{1} \mathrm{H}-{ }^{1} \mathrm{H}$ COSY spectra of 1 revealed the presence of two same sets of conjugated trans-dienes at $\delta_{\mathrm{H}} 5.69\left(1 \mathrm{H}, \mathrm{d}, J=15.4 \mathrm{~Hz}, \mathrm{H}-2, \mathrm{H}-2^{\prime}\right), 6.99(1 \mathrm{H}, \mathrm{dd}, J=15.3,11.2 \mathrm{~Hz}, \mathrm{H}-3$, H-3'), $6.14\left(1 \mathrm{H}, \mathrm{dd}, J=15.1,11.3 \mathrm{~Hz}, \mathrm{H}-4, \mathrm{H}-4^{\prime}\right), 5.64\left(1 \mathrm{H}, \mathrm{dd}, J=15.0,9.6 \mathrm{~Hz}, \mathrm{H}-5, \mathrm{H}-5^{\prime}\right)$, together with a methoxy moiety, eleven methyl groups, thirty $\mathrm{sp}^{3}$-hybridized methines and methylenes. The ${ }^{13} \mathrm{C}-\mathrm{NMR}$ and DEPT spectra illustrated characteristic signals attributable to two carbonyls at $\delta_{C} 170.3,170.2$, two same groups of olefinic methines at $\delta_{C} 121.2,145.3,132.3,144.5$, along with two hemiketal carbon at $\delta_{C} 99.3,99.4$. Through detailed analysis of NMR data, the fragments of 1 resembled those of the known compound elaiophylin (4) (please see Supplementary Materials Table S1 for the detailed data) in the macrodiolide ring, polyketide side chains, hemiketal rings and one sugar moiety, except that the 2-deoxy-L-fucose sugar unit in 4 was replaced with a methoxyl group at $\delta_{\mathrm{H}} 3.34$ $(3 \mathrm{H}, \mathrm{s}) / \delta_{\mathrm{C}} 56.7$. The methoxyl group was attached to $\mathrm{C}-13^{\prime}$ based on the HMBC correlation (Figure 2), and thus the planar structure of $\mathbf{1}$ was identified.

The measured vicinal coupling constants of the protons and NOESY correlations (Figure S6) are observed to be closely resembled those of the known halichoblelide B [23], allowing the assignment of the relative stereochemistry of $\mathbf{1}$. Furthermore, the absolute configuration of $\mathbf{1}$ was concluded to be the same with halichoblelide B due to the nearly superimposable Cotton effects [23]. Therefore, the structure of compound 1 was determined as 13'-de-2-deoxy-L-fucose-13'-O-methylelaiophylin (Figure 2) and named as halichoblelide D following the examples of halichoblelide B and C [23]. 


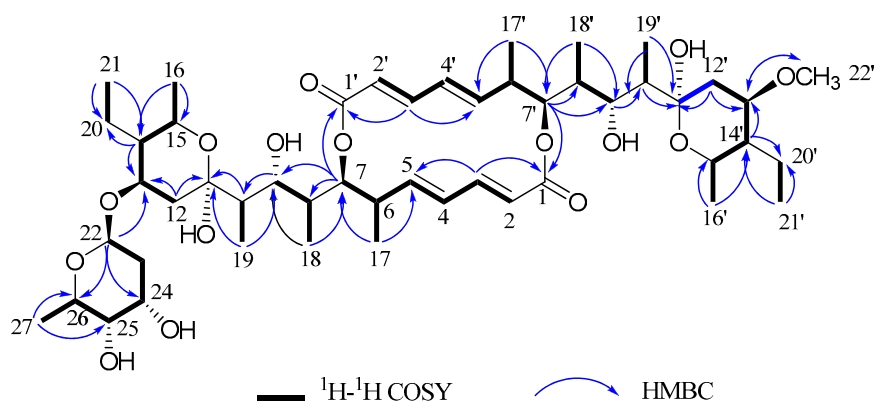

Figure 2. Selected $\mathrm{HMBC}$ and COSY correlations of compound 1 in $\mathrm{CDCl}_{3}$.

Table 1. ${ }^{13} \mathrm{C}$ - and ${ }^{1} \mathrm{H}-\mathrm{NMR}$ data $\left(500 \mathrm{MHz}, \mathrm{CDCl}_{3}\right)$ for $\mathbf{1}$.

\begin{tabular}{|c|c|c|c|c|c|}
\hline Positions & $\delta_{C}$ & $\delta_{H}(J$ in $\mathrm{Hz})$ & Positions & $\delta_{C}$ & $\delta_{\mathrm{H}}(J$ in $\mathrm{Hz})$ \\
\hline 2 & $121.2 \mathrm{~d}$ & $5.69(\mathrm{~d}, 15.4)$ & $2^{\prime}$ & $121.2 \mathrm{~d}$ & $5.69(\mathrm{~d}, 15.4)$ \\
\hline 4 & $132.3 \mathrm{~d}$ & $6.14(\mathrm{dd}, 15.1,11.3)$ & $4^{\prime}$ & $132.3 \mathrm{~d}$ & $6.14(\mathrm{dd}, 15.1,11.3)$ \\
\hline 5 & $144.5 \mathrm{~d}$ & $5.64(\mathrm{dd}, 15.0,9.6)$ & $5^{\prime}$ & $144.5 \mathrm{~d}$ & $5.64(\mathrm{dd}, 15.0,9.6)$ \\
\hline 6 & $41.0 \mathrm{~d}$ & $2.54(\mathrm{~m})$ & $6^{\prime}$ & $41.0 \mathrm{~d}$ & $2.54(\mathrm{~m})$ \\
\hline 9 & $70.9 \mathrm{~d}$ & $4.10(\mathrm{~m})$ & $9^{\prime}$ & $70.8 \mathrm{~d}$ & $4.10(\mathrm{~m})$ \\
\hline $9-\mathrm{OH}$ & & 4.12 (overlapped) & & & 4.12 (overlapped) \\
\hline 10 & $41.8 \mathrm{~d}$ & $1.70(\mathrm{t}, 7.5)$ & $10^{\prime}$ & $41.8 \mathrm{~d}$ & $1.70(\mathrm{t}, 7.5)$ \\
\hline 11 & $99.3 \mathrm{~s}$ & - & $11^{\prime}$ & $99.4 \mathrm{~d}$ & - \\
\hline $11-\mathrm{OH}$ & & $5.30(\mathrm{~d}, 2.0)$ & & & $5.23(\mathrm{~d}, 1.9)$ \\
\hline 15 & $66.7 \mathrm{~d}$ & $3.91(\mathrm{~m})$ & $15^{\prime}$ & $67.3 \mathrm{~d}$ & $3.85(\mathrm{~m})$ \\
\hline 16 & $19.5 \mathrm{q}$ & $1.09(\mathrm{~d}, 6.2)$ & $16^{\prime}$ & $19.4 \mathrm{q}$ & $1.08(\mathrm{~d}, 6.3)$ \\
\hline 17 & $15.1 \mathrm{q}$ & $1.03(\mathrm{~d}, 6.6)$ & $17^{\prime}$ & $15.1 \mathrm{q}$ & $1.03(\mathrm{~d}, 6.6)$ \\
\hline 18 & $8.9 \mathrm{q}$ & $0.81(\mathrm{~d}, 6.8)$ & $18^{\prime}$ & $8.9 \mathrm{q}$ & $0.81(\mathrm{~d}, 6.8)$ \\
\hline 19 & $7.3 \mathrm{q}$ & $0.99(\mathrm{~d}, 7.2)$ & $19^{\prime}$ & $7.3 \mathrm{q}$ & $0.99(\mathrm{~d}, 7.2)$ \\
\hline 20 & $19.6 \mathrm{t}$ & $1.43(\mathrm{~m}) ; 1.60(\mathrm{~m})$ & $20^{\prime}$ & $19.6 \mathrm{t}$ & $1.43(\mathrm{~m}) ; 1.60(\mathrm{~m})$ \\
\hline 21 & $9.3 \mathrm{q}$ & $0.83(\mathrm{t}, 7.5)$ & $21^{\prime}$ & $10.2 \mathrm{q}$ & $0.86(t, 7.5)$ \\
\hline 22 & $93.4 \mathrm{~d}$ & $5.03(\mathrm{t}, 2.1)$ & $13^{\prime}-\mathrm{OCH}_{3}$ & $56.7 \mathrm{q}$ & $3.34(\mathrm{~s})$ \\
\hline 23 & $33.8 \mathrm{t}$ & $1.78(\mathrm{dd}, 8.6,2.4)$ & & & \\
\hline 24 & $66.1 \mathrm{~d}$ & 3.97 (m) & & & \\
\hline 25 & $71.7 \mathrm{~d}$ & 3.60 (br s) & & & \\
\hline
\end{tabular}

Seven known metabolites were also isolated in this investigation and respectively identified as 2-methyl-11,11'-O-dimethylelaiophylin (2) [24], 2-methylelaiophylin (3) [24], elaiophylin (4) [11,19], 11-O-methylelaiophylin (5) [16], 11,11'-O-dimethylelaiophylin (6) [16], efomycin G (7) [14] and $11^{\prime}, 12^{\prime}$-dehydroelaiophylin (8) [17] by comparing their spectral data with the reported literature.

\subsection{Yield of Elaiophylin in Shake-Flask Fermentation under Laboratory Conditions}

Strain 219807 has been cultured on 18 different media available in our lab, and was found to produce the highest yield of elaiophylin on DO medium (50 mL in $250 \mathrm{~mL}$ Erlenmeyer flask), the yield of elaiophylin (4) measured by HPLC was calculated based on its standard curve [25]. The regression equation of elaiophylin was obtained as $y=630 x-1.6007$, and the calibration curve showed good linearity $\left(R^{2}=0.9993\right)$ (Figure S11). According to the equation, the yield of elaiophylin reached $4486 \mathrm{mg} / \mathrm{L}$ in shake-flask fermentation under laboratory conditions, which was significantly more ( $>2$ fold) than the highest yield reported before [26]. This high yield was primarily dependent on 
the strain of the microorganism. The proper medium DO which contained a complex carbon sources as observed by other reports $[27,28]$ also contributed to the high production.

\subsection{Cytotoxic Activity of Compounds $\mathbf{1}-\mathbf{7}$}

Compounds 1-7 showed potent activities against human cervical carcinoma HeLa and breast cancer MCF-7 cell lines with $\mathrm{IC}_{50}$ values ranging from 0.19 to $2.12 \mu \mathrm{M}$ (Table 2). Remarkably, the new compound 1 exhibited cytotoxic activities against HeLa and MCF-7 cell lines with $\mathrm{IC}_{50}$ values of 0.30 and $0.33 \mu \mathrm{M}$, respectively. Compound 8 was not evaluated in cytotoxicity assays for the scarcity of material.

Table 2. Cytotoxicities of compounds 1-7 against HeLa and MCF-7 cells.

\begin{tabular}{ccc}
\hline \multirow{2}{*}{ Compounds } & \multicolumn{2}{c}{ IC $_{50}(\mu \mathbf{M})$} \\
\cline { 2 - 3 } & HeLa & MCF-7 \\
\hline 1 & 0.3 & 0.33 \\
2 & 1.12 & 2.12 \\
3 & 0.29 & 0.29 \\
4 & 0.29 & 0.19 \\
$4-6^{\text {a }}$ & 0.57 & 0.96 \\
7 & 0.59 & 0.79 \\
$5-F l u o r o u r a c i l$ & 770 & 770 \\
\hline
\end{tabular}

a Compounds 4, 5 and $\mathbf{6}$ could partly interconvert when dissolved in $\mathrm{MeOH}$ [24].

\section{Experimental Section}

\subsection{General Procedures}

Optical rotations were measured using a 341 polarimeter (Perkin-Elmer, Norwalk, CT, USA). UV spectra were recorded on a UV-2600 UV-VIS spectrophotometer (Shimadzu, Tokyo, Japan). IR spectra were obtained from Nicolet Nexus 470 FT-IR spectrometer (Thermo Fisher Scientific, Waltham, MA, USA). 1D and 2D NMR spectra were determined with AM-400 and DRX-500 spectrometers (Bruker, Ettlingen, Germany) using TMS as the internal standard. Circular dichroism spectra were recorded with a Chirascan spectropolarimeter (Applied Photophysics, Leatherhead, UK). High-resolution mass spectrometric (HRMS) data were measured on a linear trapquadrupole (LTQ)-Orbitrap Velos instrument (Thermo Fisher Scientific). Analytical HPLC was carried out on a Waters 2998 (Waters, Milford, MA, USA) using a Phenomenex Gemini C18 column $(250 \mathrm{~mm} \times 4.6 \mathrm{~mm}, 5 \mu \mathrm{m}$; Phenomenex, Torrance, CA, USA). The semi-preparative HPLC instrument used (Agilent 1260 Infinity, Agilent Technologies, Santa Clara, CA, USA) was equipped with an Agilent Zorbax SB-C18 column $(250 \mathrm{~mm} \times 9.4 \mathrm{~mm}, 5 \mu \mathrm{m})$. Thin-layer chromatography was conducted on precoated silica gel 60 GF254 plates (Qingdao Haiyang Chemical, Qingdao, China) and column chromatography was performed using silica gel (200-300 mesh; Qingdao Haiyang Chemical). 3-(4,5-dimethylthiazol-z-yl)-2,5-diphenyltetrazolium bromide (Amresco, Cleveland, OH, USA) and 5-fluorouracil (Aladdin, Shanghai, China) were employed for cytotoxicity assays. An Infinite M200 Pro microplate reader (Tecan, Mannedorf, Switzerland) was used for measuring the absorbance.

\subsection{Material and Fermentation}

The strain 219807 was isolated from mangrove soil collected in Sanya, Hainan Province, China and characterized as Streptomyces sp. by $16 \mathrm{~S}$ rRNA sequence (GenBank accession number HQ992731). A voucher specimen was deposited in the Key Laboratory of Combinatorial Biosynthesis and Drug Discovery (Wuhan University, Wuhan, China), Ministry of Education, and Wuhan University School 
of Pharmaceutical Sciences (http://pharmacy.whu.edu.cn/NewsDetail.asp?MaxSort=xygk\&MaxUrl= about\&id=450) and the China Center for Type Culture Collection (CCTCC No: M 2015276).

The strain was pre-cultured on ISP2 agar medium ( $0.4 \%$ glucose, $0.4 \%$ yeast extract, $1 \%$ malt extract, pH 7.0, supplemented with $2 \%$ agar) for 5-7 days. A single colony was inoculated into $50 \mathrm{~mL}$ of ISP2 liquid medium in $250 \mathrm{~mL}$ Erlenmeyer flasks on a rotatory shaker $(220 \mathrm{rpm})$ at $28^{\circ} \mathrm{C}$ for 3 days. Subsequently, $10 \mathrm{~mL}$ of seed culture was inoculated to $1 \mathrm{~L}$ Erlenmeyer flask containing $250 \mathrm{~mL}$ of DO fermentation medium ( $1 \%$ glucose, $2.5 \%$ dextrin, $2 \%$ oatmeal, $1 \%$ cottonseed flour, $0.5 \%$ fish meal, $0.2 \%$ yeast extract, $0.3 \% \mathrm{CaCO}_{3}, \mathrm{pH}$ 6.0). Total $5 \mathrm{~L}$ of the fermentation broth was incubated for 8 days at $220 \mathrm{rpm}$ and $28^{\circ} \mathrm{C}$. The highest yield of elaiophylin (4) was obtained while using $50 \mathrm{~mL}$ liquid culture of DO medium in $250 \mathrm{~mL}$ flask, observed by HPLC and calculated based on its standard curve [25].

\subsection{Extraction and Isolation}

The fermentation broth was separated into the mycelium and supernatant after centrifuging at $12,000 \times g$ for $1 \mathrm{~h}$ at $4{ }^{\circ} \mathrm{C}$. The mycelium was disrupted by $80 \%$ acetone aqueous solution through ultrasonication for three times and evaporated under reduced pressure to yield $10.0 \mathrm{~g}$ of crude extract. The extract was subjected to silica gel column chromatography using gradient elution with cyclohexane and acetone mixture $(v / v, 90: 10-0: 100)$ to afford nine fractions: A1-A9. Fraction A5 was purified by reversed-phase HPLC $(3 \mathrm{~mL} / \mathrm{min}$, UV detection at $252 \mathrm{~nm})$ with a gradient of $\mathrm{CH}_{3} \mathrm{CN}(\mathrm{A}) / \mathrm{H}_{2} \mathrm{O}$ (B) (0-5 min, 65\% A; 5-6 min, 65\%-80\% A; 6-14 min, 80\% A; $14-15 \mathrm{~min}, 80 \%-95 \%$ A; $15-19 \mathrm{~min}$, 95\% A; $19-20 \mathrm{~min}, 95 \%-100 \%$ A; $20-23 \mathrm{~min}, 100 \% \mathrm{~A} ; 23-25 \mathrm{~min}, 65 \% \mathrm{~A})$, to yield $1(2.8 \mathrm{mg}$, $t_{R} 23.6$ min). Fraction A7 was purified by reversed-phase HPLC with a gradient of $\mathrm{CH}_{3} \mathrm{CN}(\mathrm{A}) / \mathrm{H}_{2} \mathrm{O}$ (B) (0-12 $\mathrm{min}, 79 \%$ A; $12-13 \mathrm{~min}, 79 \%-90 \%$ A; $13-29 \mathrm{~min}, 90 \%$ A; $29-30 \mathrm{~min}, 90 \%-79 \%$ A; 30-38 min, $79 \% \mathrm{~A})$ to afford six subfractions A7A-A7F. Pure compounds 4 (75.3 mg, $\left.t_{R} 5.5 \mathrm{~min}\right), 5(170.2 \mathrm{mg}$, $t_{R} 14.1 \mathrm{~min}$ ) and $6\left(386.2 \mathrm{mg}, t_{R} 34.0 \mathrm{~min}\right)$ were directly obtained from A7A, A7B and A7E, respectively. Subfraction A7C was further purified by reverse-phase HPLC eluted with $\mathrm{CH}_{3} \mathrm{CN} / \mathrm{H}_{2} \mathrm{O}$ (64:36) to give 8 ( $\left.2.8 \mathrm{mg}, t_{R} 10.0 \mathrm{~min}\right)$. Subfraction A7D was separated by reverse-phase HPLC with an isocratic solvent system of $\mathrm{CH}_{3} \mathrm{CN} / \mathrm{H}_{2} \mathrm{O}$ (53:47) to obtain 7 (15.2 mg, $\left.t_{R} 10.5 \mathrm{~min}\right)$. Subfraction A7F was subjected to reversed-phase HPLC with a gradient of $\mathrm{CH}_{3} \mathrm{CN}(\mathrm{A}) / \mathrm{H}_{2} \mathrm{O}$ (B) $(0-4 \mathrm{~min}, 80 \% \mathrm{~A} ; 4-5 \mathrm{~min}$, 80\%-87\% A; 5-12 $\mathrm{min}, 87 \%$ A; $12-13 \mathrm{~min}, 87 \%-99 \%$ A; $13-25 \mathrm{~min}, 99 \%$ A; $25-26 \mathrm{~min}, 99 \%-80 \%$ A; $26-30 \mathrm{~min}, 80 \% \mathrm{~A})$ to afford $3\left(5.1 \mathrm{mg}, t_{R} 13.1 \mathrm{~min}\right)$ and $2\left(15.2 \mathrm{mg}, t_{R} 28.4 \mathrm{~min}\right)$.

13'-De-2-deoxy-L-fucose-13'-O-methylelaiophylin (Halichoblelide D, 1): White needle-like crystals (2.8 mg), $[\alpha]_{\mathrm{D}}^{20}-3.36(\mathrm{c} 0.055, \mathrm{MeOH}) ; \mathrm{UV}(\mathrm{MeOH}) \lambda \max (\log \varepsilon) 252(4.29) \mathrm{nm} ; \mathrm{IR}\left(\nu_{\max } \mathrm{cm}^{-1}\right) 3523,3445,3333$, 2977, 2931, 1699, 1636, 1458, 1382, 1302, 1222, 1184, 1088, 981, 807, 743, 695, 641, 567; CD (MeOH) 213 $(\Delta \varepsilon-14.2), 250(\Delta \varepsilon-34.9), 279(\Delta \varepsilon+23.9)$; HRESIMS: $m / z 943.5183[\mathrm{M}+\mathrm{Cl}]^{-}$(calc. for $\mathrm{C}_{49} \mathrm{H}_{80} \mathrm{O}_{15} \mathrm{Cl}^{-}$, 943.5186). ${ }^{1} \mathrm{H}-\mathrm{NMR}\left(500 \mathrm{MHz}, \mathrm{CDCl}_{3}\right) \delta 5.69(1 \mathrm{H}, \mathrm{d}, J=15.4 \mathrm{~Hz}, \mathrm{H}-2), 6.99(1 \mathrm{H}, \mathrm{dd}, J=15.3$, $11.2 \mathrm{~Hz}, \mathrm{H}-3), 6.14(1 \mathrm{H}, \mathrm{dd}, J=15.1,11.3 \mathrm{~Hz}, \mathrm{H}-4), 5.64(1 \mathrm{H}, \mathrm{dd}, J=15.0,9.6 \mathrm{~Hz}, \mathrm{H}-5), 2.54(1 \mathrm{H}$, m, H-6), $4.72(1 \mathrm{H}, \mathrm{d}, J=10.3 \mathrm{~Hz}, \mathrm{H}-7), 1.95(1 \mathrm{H}, \mathrm{m}, \mathrm{H}-8), 4.10(1 \mathrm{H}, \mathrm{m}, \mathrm{H}-9), 4.12(1 \mathrm{H}$, overlapped, 9-OH), $1.70(1 \mathrm{H}, \mathrm{t}, J=7.5 \mathrm{~Hz}, \mathrm{H}-10), 5.30(1 \mathrm{H}, \mathrm{d}, J=2.0 \mathrm{~Hz}, 11-\mathrm{OH}), 1.00(1 \mathrm{H}, \mathrm{m}, \mathrm{H}-12 \mathrm{a}), 2.37(1 \mathrm{H}, \mathrm{dd}, J=11.9$, $4.5 \mathrm{~Hz}, \mathrm{H}-12 \mathrm{~b}), 3.95(1 \mathrm{H}, \mathrm{m}, \mathrm{H}-13), 1.18(1 \mathrm{H}, \mathrm{m}, \mathrm{H}-14), 3.91(1 \mathrm{H}, \mathrm{m}, \mathrm{H}-15), 1.09$ (3H, d, J = 6.2 Hz, H-16), $1.03(3 \mathrm{H}, \mathrm{d}, J=6.6 \mathrm{~Hz}, \mathrm{H}-17), 0.81(3 \mathrm{H}, \mathrm{d}, J=6.8 \mathrm{~Hz}, \mathrm{H}-18), 0.99(3 \mathrm{H}, \mathrm{d}, J=7.2 \mathrm{~Hz}, \mathrm{H}-19), 1.43(1 \mathrm{H}, \mathrm{m}$, H-20a); 1.60 (1H, m, H-20b), $0.83(3 \mathrm{H}, \mathrm{t}, J=7.5 \mathrm{~Hz}, \mathrm{H}-21), 5.03(1 \mathrm{H}, \mathrm{t}, J=2.1 \mathrm{~Hz}, \mathrm{H}-22), 1.78(2 \mathrm{H}, \mathrm{dd}$, $J=8.6,2.4 \mathrm{~Hz}, \mathrm{H}-23), 3.97(1 \mathrm{H}, \mathrm{m}, \mathrm{H}-24), 3.60(1 \mathrm{H}, \mathrm{br} \mathrm{s}, \mathrm{H}-25), 3.98(1 \mathrm{H}, \mathrm{m}, \mathrm{H}-26), 1.23(3 \mathrm{H}, \mathrm{d}$, $J=6.6 \mathrm{~Hz}, \mathrm{H}-27), 5.69\left(1 \mathrm{H}, \mathrm{d}, J=15.4 \mathrm{~Hz}, \mathrm{H}-2^{\prime}\right), 6.99\left(1 \mathrm{H}, \mathrm{dd}, J=15.3,11.2 \mathrm{~Hz}, \mathrm{H}-3^{\prime}\right), 6.14(1 \mathrm{H}, \mathrm{dd}$, $\left.J=15.1,11.3 \mathrm{~Hz}, \mathrm{H}-4^{\prime}\right), 5.64\left(1 \mathrm{H}, \mathrm{dd}, J=15.0,9.6 \mathrm{~Hz}, \mathrm{H}-5^{\prime}\right), 2.54\left(1 \mathrm{H}, \mathrm{m}, \mathrm{H}-6^{\prime}\right), 4.72(1 \mathrm{H}, \mathrm{d}, J=10.3 \mathrm{~Hz}$, H-7'), $1.95\left(1 \mathrm{H}, \mathrm{m}, \mathrm{H}-8^{\prime}\right), 4.10\left(1 \mathrm{H}, \mathrm{m}, \mathrm{H}-9^{\prime}\right), 4.12\left(1 \mathrm{H}\right.$, overlapped, $\left.9^{\prime}-\mathrm{OH}\right), 1.70\left(1 \mathrm{H}, \mathrm{t}, J=7.5 \mathrm{~Hz}, \mathrm{H}-10^{\prime}\right)$, $5.23\left(1 \mathrm{H}, \mathrm{d}, J=1.9 \mathrm{~Hz}, 1^{\prime}-\mathrm{OH}\right), 1.00\left(1 \mathrm{H}, \mathrm{m}, \mathrm{H}-12^{\prime} \mathrm{a}\right), 2.45\left(1 \mathrm{H}, \mathrm{dd}, J=12.0,4.5 \mathrm{~Hz}, \mathrm{H}-12^{\prime} \mathrm{b}\right), 3.47(1 \mathrm{H}$, $\left.\mathrm{td}, J=10.7,4.7 \mathrm{~Hz}, \mathrm{H}-13^{\prime}\right), 1.13\left(1 \mathrm{H}, \mathrm{m}, \mathrm{H}-14^{\prime}\right), 3.85\left(1 \mathrm{H}, \mathrm{m}, \mathrm{H}-15^{\prime}\right), 1.08\left(3 \mathrm{H}, \mathrm{d}, J=6.3 \mathrm{~Hz}, \mathrm{H}-16^{\prime}\right), 1.03$ $\left(3 \mathrm{H}, \mathrm{d}, J=6.6 \mathrm{~Hz}, \mathrm{H}-17^{\prime}\right), 0.81\left(3 \mathrm{H}, \mathrm{d}, J=6.8 \mathrm{~Hz}, \mathrm{H}-18^{\prime}\right), 0.99\left(3 \mathrm{H}, \mathrm{d}, J=7.2 \mathrm{~Hz}, \mathrm{H}-19^{\prime}\right), 1.43(1 \mathrm{H}$, $\left.\mathrm{m}, \mathrm{H}-2 \mathrm{O}^{\prime} \mathrm{a}\right) ; 1.60\left(1 \mathrm{H}, \mathrm{m}, \mathrm{H}-2 \mathrm{O}^{\prime} \mathrm{b}\right), 0.86\left(3 \mathrm{H}, \mathrm{t}, J=7.5 \mathrm{~Hz}, \mathrm{H}-21^{\prime}\right), 3.34\left(3 \mathrm{H}, \mathrm{s}, 13^{\prime}-\mathrm{OCH}_{3}\right) ;{ }^{13} \mathrm{C}-\mathrm{NMR}$ 
$\left(125 \mathrm{MHz}, \mathrm{CDCl}_{3}\right) \delta 170.3(\mathrm{C}-1), 170.2\left(\mathrm{C}-1^{\prime}\right), 121.2\left(\mathrm{C}-2,2^{\prime}\right), 145.3\left(\mathrm{C}-3, \mathrm{C}-3^{\prime}\right), 132.3\left(\mathrm{C}-4, \mathrm{C}-4^{\prime}\right), 144.5$ $\left(\mathrm{C}-5, \mathrm{C}-5^{\prime}\right), 41.0\left(\mathrm{C}-6, \mathrm{C}-6^{\prime}\right), 78.1(\mathrm{C}-7), 77.4\left(\mathrm{C}-7^{\prime}\right), 36.1$ (C-8, C-8'), $70.9(\mathrm{C}-9), 70.8\left(\mathrm{C}-9^{\prime}\right), 41.8$ (C-10, C-10'), 99.3 (C-11), $99.4\left(\mathrm{C}-11^{\prime}\right), 39.1$ (C-12, C-12'), 70.4 (C-13), $76.1\left(\mathrm{C}-13^{\prime}\right), 56.7$ (13'-OCH3), 48.6 (C-14), $49.3\left(\mathrm{C}-14^{\prime}\right), 66.7(\mathrm{C}-15), 67.3\left(\mathrm{C}-15^{\prime}\right), 19.5(\mathrm{C}-16), 19.4\left(\mathrm{C}-16^{\prime}\right), 15.1\left(\mathrm{C}-17, \mathrm{C}-17^{\prime}\right), 8.9\left(\mathrm{C}-18, \mathrm{C}-18^{\prime}\right)$, $7.3\left(\mathrm{C}-19, \mathrm{C}-19^{\prime}\right), 19.6\left(\mathrm{C}-20, \mathrm{C}-20^{\prime}\right), 9.3$ (C-21), 10.2 (C-21'), $93.4(\mathrm{C}-22), 33.8$ (C-23), 66.1 (C-24), 71.7 (C-25), 66.3 (C-26), 17.0 (C-27).

\subsection{Cytotoxic Activity Assay}

Compounds 1-7 were evaluated for cytotoxic effects against HeLa and MCF-7 cells according to the previously described MTT method [29],. The absorbance at $570 \mathrm{~nm}$ was determined on a Tecan Infinite M200 Pro reader, and the reference wavelength was $690 \mathrm{~nm}$. The experiment was conducted in triplicate. 5-Fluorouracil was employed as positive control.

\section{Conclusions}

Eight a group of elaiophylins comprised of a new derivative, halichoblelide D (1), together with seven known compounds 2-8 was isolated and identified from mangrove-derived Streptomyces sp. 219807. Compounds 1-7 displayed significant cytotoxic activities against HeLa and MCF-7 cells with $\mathrm{IC}_{50}$ values $0.19-2.12 \mu \mathrm{M}$, and were thus promising candidates for future development of antineoplastic drugs. Notably, the strain possesses the capacity of producing elaiophylin (4) reaching the hitherto highest yield $(4486 \mathrm{mg} / \mathrm{L})$ reported at lab scale.

Supplementary Materials: The UV, IR, CD, NMR and HRMS spectra of compound 1, and a standard curve of elaiophylin are available online at: http://www.mdpi.com/1420-3049/21/8/970.

Acknowledgments: This work was partially supported by the EU FP7 project PharmaSea (312184) and the China "973" program (2012CB721001). The authors are grateful to Marcel Jaspars and Hai Deng, Rainer Ebel and Jioji Tabudravu in Marine Biodiscovery Centre, Department of Chemistry, University of Aberdeen, for their help on HPLC-HRESIMS and analysis in the database AntiBase 2012.

Author Contributions: Kui Hong and Zixin Deng conceived and designed the experiments; Ying Han and Erli Tian performed the experiments and analyzed the data; Dongbo Xu and Min Ma contributed reagents and analysis tools; Erli Tian and Ying Han wrote the paper.

Conflicts of Interest: The authors declare no conflict of interest.

\section{References}

1. Davies, J.; Davies, D. Origins and evolution of antibiotic resistance. Microbiol. Mol. Biol. Rev. 2010, 74, 417-433. [CrossRef] [PubMed]

2. Penesyan, A.; Gillings, M.; Paulsen, I.T. Antibiotic discovery: Combatting bacterial resistance in cells and in biofilm communities. Molecules 2015, 20, 5286-5298. [CrossRef] [PubMed]

3. Demain, A.L.; Sanchez, S. Microbial drug discovery: 80 years of progress. J. Antibiot. 2009, 62, 5-16. [CrossRef] [PubMed]

4. Xu, D.B.; Ye, W.W.; Han, Y.; Deng, Z.X.; Hong, K. Natural Products from Mangrove Actinomycetes. Mar. Drugs 2014, 12, 2590-2613. [CrossRef] [PubMed]

5. Wang, P.; Kong, F.D.; Wei, J.J.; Wang, Y.; Wang, W.; Hong, K.; Zhu, W.M. Alkaloids from the mangrove-derived actinomycete Jishengella endophytica 161111. Mar. Drugs 2014, 12, 477-490. [CrossRef] [PubMed]

6. Li, X.L.; Xu, M.J.; Zhao, Y.L.; Xu, J. A novel benzo[f][1,7]naphthyridine produced by Streptomyces albogriseolus from mangrove sediments. Molecules 2010, 15, 9298-9307. [CrossRef] [PubMed]

7. Kathiresan, K.; Bingham, B.L. Biology of mangroves and mangrove ecosystems. Adv. Mar. Biol. 2001, 40, 81-251.

8. Feling, R.H.; Buchanan, G.O.; Mincer, T.J.; Kauffman, C.A.; Jensen, P.R.; Fenical, W. Salinosporamide A: A highly cytotoxic proteasome inhibitor from a novel microbial source, a marine bacterium of the new genus Salinospora. Angew. Chem. Int. Ed. 2003, 42, 355-357. [CrossRef] [PubMed]

9. Ding, L.; Munch, J.; Goerls, H.; Maier, A.; Fiebig, H.H.; Lin, W.H.; Hertweck, C. Xiamycin, a pentacyclic indolosesquiterpene with selective anti-HIV activity from a bacterial mangrove endophyte. Bioorg. Med. Chem. Lett. 2010, 20, 6685-6687. [CrossRef] [PubMed] 
10. Fu, P.; Yang, C.; Wang, Y.; Liu, P.; Ma, Y.; Xu, L.; Su, M.; Hong, K.; Zhu, W. Streptocarbazoles A and B, two novel indolocarbazoles from the marine-derived actinomycete strain Streptomyces sp. FMA. Org. Lett. 2012, 14, 2422-2425. [CrossRef] [PubMed]

11. Arcamone, F.M.; Bertazzoli, C.; Ghione, M.; Scotti, T.G. Melanosporin and elaiophylin, new antibiotics from Streptomyces melanosporus (sive melanosporofaciens) n. sp. Microbiology 1959, 7, 207-216.

12. Arai, M. Azalomycins B and F, two new antibiotics II. Properties and isolation. J. Antibiot. 1960, 13, 46-50. [PubMed]

13. Fiedler, H.P.; Worner, W.; Zahner, H.; Kaiser, H.P.; Keller-Schierlein, W.; Muller, A. Metabolic products of microorganisms. 200. Isolation and characterisation of niphithricins $\mathrm{A}$ and $\mathrm{B}$, and elaiophylin, antibiotics produced by Streptomyces violaaceoniger. J. Antibiot. 1981, 34, 1107-1118. [CrossRef] [PubMed]

14. Cui, C.B.; Wang, H.; Han, B.; Song, Y.X. Elaiophylins, new cell cycle inhibitors and apoptosis inducers, produced by Streptomyces pseudoverticillus (III). Structures and NMR studies. Chin. J. Med. Chem. 2001, 11, $25-31$.

15. Grabley, S.; Hammann, P.; Raether, W.; Wink, J.; Zeeck, A. Secondary metabolites by chemical screening. II. Amycins A and B, two novel niphimycin analogs isolated from a high producer strain of elaiophylin and nigericin. J. Antibiot. 1990, 43, 639-647. [CrossRef] [PubMed]

16. Ritzau, M.; Heinze, S.; Fleck, W.F.; Dahse, H.M.; Gräfe, U. New macrodiolide antibiotics, 11-O-monomethyl-and 11,11'-O-dimethylelaiophylins, from Streptomyces sp. HKI-0113 and HKI-0114. J. Nat. Prod. 1998, 61, 1337-1339. [CrossRef] [PubMed]

17. Wu, C.Y.; Tan, Y.; Gan, M.L.; Wang, Y.G.; Guan, Y.; Hu, X.X.; Zhou, H.X.; Shang, X.Y.; You, X.F.; Yang, Z.Y.; Xiao, C.L. Identification of elaiophylin derivatives from the marine-derived actinomycete Streptomyces sp. 7-145 using PCR-based screening. J. Nat. Prod. 2013, 76, 2153-2157. [CrossRef] [PubMed]

18. Gerlitz, M.; Hammann, P.; Thiericke, R.; Rohr, J. The biogenetic origin of the carbon skeleton and the oxygen atoms of elaiophylin, a symmetric macrodiolide antibiotic. J. Org. Chem. 1992, 57, 4030-4033. [CrossRef]

19. Lee, S.Y.; Kim, M.S.; Kim, H.S.; Kim, Y.H.; Hong, S.D.; Lee, J.J. Structure determination and biological activities of elaiophylin produced by Streptomyces sp. MCY-846. J. Microbiol. Biotechnol. 1996, 6, 245-249.

20. Doseung, L.; Woo, J.K.; Kim, D.; Kim, M.; Cho, S.K.; Kim, J.H.; Park, S.P.; Lee, H.Y.; Riu, K.Z.; Lee, D.S. Antiviral activity of methylelaiophylin, an $\alpha$-glucosidase inhibitor. J. Microbiol. Biotechnol. 2011, 21, $263-266$.

21. Lee, S.Y.; Kim, H.S.; Kim, Y.H.; Kim, S.B.; Han, S.B.; Kim, H.M.; Hong, S.D.; Lee, J.J. Immunosupressive activity of elaiophylins. J. Microbiol. Biotechnol. 1997, 7, 272-277.

22. Takahashi, S.; Arai, M.; Ohki, E. Chemical studies on azalomycins. I. Preliminary study on azalomycin-B. Chem. Pharm. Bull. 1967, 15, 1651-1656. [CrossRef] [PubMed]

23. Yamada, T.; Kikuchi, T.; Tanaka, R.; Numata, A. Halichoblelides B and C, potent cytotoxic macrolides from a Streptomyces species separated from a marine fish. Tetrahedron Lett. 2012, 53, 2842-2846. [CrossRef]

24. Sheng, Y.; Lam, P.W.; Shahab, S.; Santosa, D.A.; Proteau, P.J.; Zabriskie, T.M.; Mahmud, T. Identification of elaiophylin skeletal variants from the indonesian Streptomyces sp. ICBB 9297. J. Nat. Prod. 2015, 78, 2768-2775. [CrossRef] [PubMed]

25. Hong, K.; Han, Y.; Tian, E.L.; Ma, M.; Deng, Z.X. Streptomyces for Manufacture of Elaiophylin Compounds as Antitumors. CN 104876984A. 2 September 2015.

26. Jiang, S.; Tao, J.H.; Zhu, L.; Huang, W.Y. Regulation of short chain fatty acids on azalomycin B biosynthesis. Chin. J. Antibiot. 2009, 34, 219-223.

27. Gesheva, V.; Ivanova, V.; Gesheva, R. Effects of nutrients on production of antifungal AK-111-81 macrolide antibiotic. Microbiol. Res. 2005, 160, 243-248. [CrossRef] [PubMed]

28. Ilić, S.B.; Konstantinović, S.S.; Cvijović, G.Đ.G.; Savić, D.S.; Veljković, V.B. The impact of glycerol and some carbohydrates on antibiotic production by Streptomyces hygroscopicus CH-7. Med. Chem. Res. 2013, 22, 934-937. [CrossRef]

29. Xu, D.B.; Ma, M.; Liu, Y.F.; Zhou, T.; Wang, K.X.; Deng, Z.X.; Hong, K. PreQ 0 base, an unusual metabolite with anti-cancer activity from Streptomyces qinglanensis 172205. Anticancer Agents Med. Chem. 2015, 15, 285-290. [CrossRef] [PubMed]

Sample Availability: Sample of the compound 4 is available from the authors.

(C) 2016 by the authors; licensee MDPI, Basel, Switzerland. This article is an open access article distributed under the terms and conditions of the Creative Commons Attribution (CC-BY) license (http://creativecommons.org/licenses/by/4.0/). 